\title{
The promise of nanoneuromedicine
}

\begin{abstract}
"While biomaterials, sensors, nanoparticles, and nanospheres are potential platforms for drug and gene delivery for nanoneuromedicines, few are fully developed for human use, and each hold a number of challenges."
\end{abstract}

\section{KEYWORDS: drug targeting " hyperthermia $\approx$ magnetic nanoparticles $\approx$ MRI - nanotoxicity}

The American Society for Nanomedicine (ASNM) has long served as a platform for exchange of seminal research findings. A focus on translation, from bench to bedside forms the society's unique signature. The ASNM was founded as a registered nonprofit, professional society with a membership base drawn from diverse fields of engineering, medicine, law, government policy, ethics, toxicology, biotechnology and pharmaceutical sciences. Speedy clinical translation of inventions is fostered. Nanoneuromedicine, the conference theme for 2014, seeks to best define an evolving field by bringing divergent disciplines and approaches together in discussion in order to facilitate longterm collaborative research. The efforts will serve as a catalyst for product development from the laboratory bench to human clinical trials.

\section{Defining nanoneuromedicine}

In initiating the conference, an initial goal was to identify the components that fill the whole of nanoneuromedicine. Research activities focused on the development of effective treatments for many nervous system maladies and focusing the conference to specific areas proved challenging. What was clear, however, was the directive towards improving nervous system function or halting disease. Neurodegenerative, addictive, infectious and neuroinflammatory disorders continue to be substantive and unabated contributions to human suffering. While biomaterials, sensors, nanoparticles, and nanospheres are potential platforms for drug and gene delivery for nanoneuromedicines, few are fully developed for human use, and each hold a number of challenges [1]. A few examples are noteworthy and include, but are not limited to, preventing aggregation of misfolded proteins, limiting toxicities of growth and immune modulatory factors, inducing neural repair, improving diagnostics, implementing neuroprotective nanosurgeries for device implantation, and cell-mediated deliveries across a functional blood-brain barrier (BBB). Each can positively affect disease outcomes. The general field of nanomedicines includes drug formulations containing nucleic acids, proteins and antibodies that serve to improve medicine pharmacokinetics by delivering multifunctional payloads to reverse tissue damage. This is true for all applications of the technology both inside and outside the nervous system. For example, those formulations that target diseased cells can facilitate cancer cell destruction and reduce untoward secondary side effects. The technologies are applicable to a range of disorders. Liposomal formulations of doxorubicin $\left(\right.$ Doxil $\left.{ }^{\circledR}\right)$, for example, can reduce drugrelated cardiotoxicity. Coating formulations can affect drug half-lives and improve brain tumor therapeutic outcomes [2]. Targeting particles to immune cells can facilitate microbial clearance for meningitis. For example, liposomal formulations of amphotericin B (Ambisome ${ }^{\circledR}$ ) positively affect treatment outcomes following cryptococcal and Leishmania infections. In both instances the formulations hijack macrophages' scavenging activity for maximal therapeutic gain. Most importantly, this enables reduction in collateral tissue toxicities and enhanced drug efficacy during facilitated drug entry into the CNS. Similar strategies are operative for nanoformulations of antiretroviral drugs and especially those where brain entry is deemed appropriate. These cell-based therapies improve drug pharmacokinetics and pharmacodynamics, including those designed to clear viral reservoirs contained both inside and outside the nervous system.

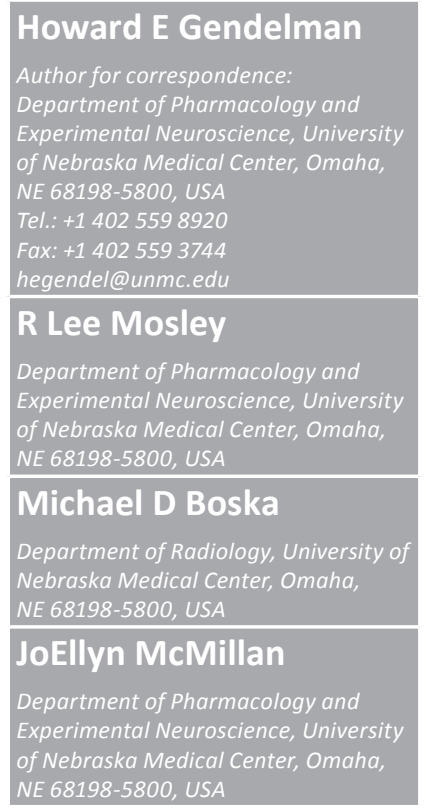

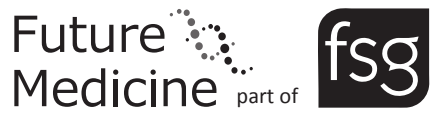




\section{Nanoneuromedicine \& bioimaging}

The field of nanoneuroscience combines both therapeutic and diagnostic disciplines. Interestingly, PET serves both diagnostic and therapeutic nanomedicine capacities (called theranostics). In stroke models, for example, nearly absent blood flow is better visualized through oxygen-carrying liposome-encapsulated hemoglobin. These can penetrate the core of ischemic zones [3]. A fibrin-specific, liquid perfluorocarbon nanoparticle with bound plasminogen activator streptokinase can provide rapid fibrinolysis [4]. Compounds capable of scavenging reactive oxygen species and providing neuroprotection include cerium oxide nanoparticles [1]. MRI also allows rapid assessment of drug pharmacokinetics and is augmented by contrast agents. The most commonly used MRI contrast agent is superparamagnetic iron oxide (SPIO), which causes a dramatic loss of signal over a spatial range of approximately 100-times the particle size. Single micron particles are detected with $100-\mu \mathrm{m}$ isotropic resolution [5]. Quantitation of particle numbers is accessible using MRI $\mathrm{T}_{2}$ relaxometry. The most common application of magnetite targeting is in cancer therapy. SPIOloaded nanoparticles can be used for imaging and targeted therapeutic delivery to brain tumors. Such formulations can access tumors after delivery of nanomaterials across the BBB [6]. 'Steerable' particles can be used in combination with carmustine, a hydrophilic polymeric coating. Accumulation of such nanoparticles in brain tumors by magnetic attraction can affect tumor volume reductions [7]. SPIO-loaded nanomaterials may facilitate cell tracking of immune cells. The design of such nanomaterials enables the particles to be taken up efficiently by cells while remaining inert. Preventing interference with cellular function is a critical property for diagnostic nanoneuromedicines. Studies range from simple polymer-coated SPIO particles, to SPIO-labeled macrophages, to strategies for labeling primary islet cells $[8]$.

Optimization of cellular uptake of nanoformulated SPIO is an active area of interest as it permits rapid evaluation of drug biodistribution and theranostics. Specific cellular uptake is affected by size, shape and targeting moieties. Various strategies include coating with antibodies, enzyme substrates and transfection vectors. In addition, size, shape, $\zeta$-potential and targeting density are combinatorial factors in the effectiveness of cell-specific particle uptake. Recent studies have led to the development of theranostic nanomaterials containing antiretroviral drugs and SPIO particles, termed 'small magnetite antiretroviral therapy' (also known as SMART particles). In these studies, drug concentration in tissue was matched by the SPIO content of the same tissue measured using MRI $\mathrm{T}_{2}$ relaxometry. Work using targeting ligands on nanoformulated SPIO particles to assess the ability of long-acting antiretroviral drugs to provide dose reductions and improve the potential for HIV eradication in the CNS is progressing [9].

\section{Targeted therapy \& toxicology of nanoneuromedicines}

A major developmental concern is how best to target the CNS while reducing general cytotoxicities. Notably, unique challenges exist for the development of effective nanotherapeutics for neuroscience. These include their ability to cross the $\mathrm{BBB}$ and maintain therapeutic drug levels in the CNS. Drugs must be effective at low concentrations and exhibit both low toxicity and low immunogenicity [1]. To surmount these challenges, a number of strategies have been employed in formulation development. Liposomal drugs can cross the BBB. Such a strategy is commonly employed for antiepileptics, -fungals, -retrovirals, -ischemics and chemotherapeutic agents. Specific targeting moieties are added on the surface to facilitate delivery to sites of action. Micelles, using amphiphilic block polymers that surround noncovalently bound drugs, can also cross the BBB [10]. Polymeric nanoparticles using biodegradable polymers to encapsulate $\operatorname{drug}(s)$ or carry it by attaching it to the nanoparticle surface can speed BBB passage by tight junctions, facilitated endocytosis or inhibition of efflux transporters on endothelial cells. Dendrimers can carry drug(s) within their own branching structures and can be modified to enhance penetration into the brain. Macrophages may also be used as drug carriers to deliver nanoparticles across the BBB [11].

"...unique challenges exist for the
development of effective nanotherapeutics
for neuroscience [including] their ability to
cross the blood-brain barrier and maintain
therapeutic drug levels in the CNS."

With the development of nanoparticles also comes concern over potential adverse effects. Special concerns have been raised over their small size, surface charge, molecular composition, surface area, route and duration 
of administration. This can and often does increase the potential for toxic cellular interactions. Notably nanoparticulate carriers can elicit pro-oxidant and -inflammatory properties. Thus, efforts to reduce potential adverse toxicities have focused on the use of biodegradable and biocompatible polymers that can evade the reticuloendothelial system and better promote nanocarrier drug release [6]. Reduction in dose and dose frequency may be achieved with the addition of cell-specific targeting moieties.

\section{Diseases of the nervous system: new opportunities for nanoneuromedicine}

Considerable efforts are being focused in the research laboratory on using nanoneuromedicine for disease treatment. For neurodegenerative disorders including Alzheimer's disease (AD) and Parkinson's disease (PD), as well as amyotrophic lateral sclerosis (ALS) and multiple sclerosis (MS), nanomedicines offer particular treatment opportunities. The particular abilities of the formulations to affect pathways linked by common pathophysiological conditions, such as neuroinflammation and misfolded proteins, which drive a cycle of degeneration, can provide improved drug targeting. The promise of nanotechnology can also help to diagnose and to monitor effects of therapeutics.

$\mathrm{AD}$ presents as impaired memory, cognition and behavior that result from brain atrophy and loss of cortical and subcortical neurons [12]. Extracellular senile plaques and intracellular neurofibrillary tangles contain aggregated amyloid- $\beta(A \beta)$, and aggregated and helical forms of hyperphosphorylated tau, respectively [13]. Diagnosis of $A D$ is focused on tau, amyloidogenic $A \beta_{42}$ forms, $A P P$ and $A \beta$-derived diffusible ligands (ADDL). ADDL levels in cerebrospinal fluid can be measured using 'biobarcodes' that utilize ADDL-specific antibodies and gold $(\mathrm{Au})$ nanoparticles [14]. Au particle conjugation to anti-A $\beta_{40}$ combined with the electrical detection system associated with scanning tunneling microscopy increases detection of the immunocomplexes [15]. Au particles also can be fabricated as multispot-localized surface plasmon resonance immunochips [16]. Nanotechnology can thus be used to detect $A \beta$ deposits. $A \beta$-coupled ultrasmall SPIO particles in conjunction with magnetic resonance microimaging ( $\mu \mathrm{MRI})$ can be used to detect $A \beta$ deposition [17]. Fluorescent quantum dots conjugated with anti-A $\beta$ antibody can track $A \beta$ accumulation [18]. Importantly, nanotherapeutics are designed to inhibit the formation of $A \beta$ aggregates. Copolymeric $N$-isopropylacrylamide/ $N$-tert-butylacrylamide particles quench and temporarily reverse fibrillization of $A \beta$ [19]. Fullerenes that inhibit $A \beta$ aggregate assembly prevent $A \beta$-mediated impairment of cognitive performance [20]. Au particles conjugated with $A \beta$ fragments can be incorporated into $A \beta$ complexes, and the $A \beta$ aggregates can be selectively ablated by laser or microwave exposures [21]. Nanotherapeutics for $\mathrm{AD}$ also include formulating $\operatorname{drug}(\mathrm{s})$ to increase brain delivery. Tacrine, one of four cholinesterase inhibitors, can be incorporated into magnetic chitosan microparticles and magnetically guided by selective placement of the magnet to the head [22]. These are but a few examples of how nanomedicines have entered into the field of neurotherapeutics in AD.

\section{"The particular abilities of the formulations to affect pathways linked by common pathophysiological conditions, such as neuroinflammation and misfolded proteins, which drive a cycle of degeneration, can provide improved drug targeting."}

PD, the second most common neurodegenerative disease, is characterized by resting tremors, bradykinesia, gait difficulties and postural instability, resulting from the loss of dopaminergic neurons within the substantia nigra pars compacta with subsequent loss of its striatal projections [23]. Proteinaceous neuronal inclusions, termed Lewy bodies, comprised chiefly of misfolded $\alpha$-synuclein $(\alpha$-syn) and ubiquitin, are disease hallmarks. For diagnosis, Au-doped $\mathrm{TiO}_{2}$ nanotube arrays are designed with a high sensitivity photoelectrochemical immunosensor to detect $\alpha$-syn [24]. To detect protein misfolding of single $\alpha$-syn molecules, nanotechnology can be paired with atomic force microscopy for enhanced detection of interprotein interactions [25]. Au particles can be interfaced with plasmon absorbance for assessment of neurotransmitter concentrations that can indirectly reflect PD pathology [26]. To combat symptoms of disease, levadopa was encased with ascorbic acid-functionalized $\mathrm{C}_{60}$ fullerenes to potentiate antioxidant activities [27]. To decrease microglia-activated reactive oxygen species, catalase was packaged into particles consisting of a block copolymer complex with polyethyleneimine-PEG, and the particles taken up by macrophages, resulting in diminished microglial-derived hydrogen peroxide, neuroinflammation and neurodegeneration in 
PD models [28]. The dopaminergic agonist bromocriptine was formulated as a particle with tristearin/tricaprin lipid combination to protect against neurodegeneration [29]. Loading dopamine into chitosan particles increased levels of striatal dopamine with limited dopamine toxicities [30]. Lactoferrin-modified particles that encapsulate the human neurotrophic factor gene were able to positively affect locomotor activity, reduce dopaminergic neuronal loss and enhance monoamine neurotransmitter levels [31]. Additionally, anti- $\alpha$-syn-conjugated polybutylcyanoacrylate particles improved intracellular $\alpha$-syn clearance [32].

ALS is a disorder of motor neurons leading to loss of neuromuscular control and eventual death resulting from respiratory failure [33]. The neuropathologic hallmark is motor neuron degeneration confined to the upper and lower motor neurons. Proteinaceous inclusions in cell bodies and axons can be found that contain ubiquitin and one or more ALS-associated proteins, such as SOD1. To facilitate the diagnosis of ALS, Au particles coated with SOD1 are used to interact with SOD1 aggregates and, as such, provide a simple and sensitive colorimetric detection system [34]. Poly(lactic-coglycolic) acid nanoparticles containing SOD1, when delivered to neurons, provided protection against hydrogen peroxide-induced oxidative stress [35]. Carboxyfullerene SOD mimetics are neuroprotective in laboratory systems of disease [36]. Nanoparticles with high loading capacity can deliver riluzole to sites of ALS pathology with greater efficacy and more accurate distribution than free riluzole [37]. MRI performed on ALS rats injected with SPIO particles conjugated with anti-CD4 antibodies revealed trafficking of immunocytes to the pathological regions [38].

In MS, the immune system attacks the selfantigens of the CNS associated with myelin and oligodendrocytes, producing lesions of demyelinated white matter or plaques with intense perivascular inflammation [39]. Nanotechnologies relevant to diagnosis in MS models have addressed the detection of inflammation. Using MRI and nanoparticles functionalized with the glycan ligand sialyl Lewis ${ }^{\mathrm{X}}$ the presence of E/P-selectins was detected in an experimental autoimmune encephlomyelitis model [40], while antibody-conjugated microparticles carrying iron oxide detected VCAM-1 [41]. By labeling macrophages with iron-oxide particles, MRI followed the migration of cells into sites of active lesion formation. Nanomedicine therapeutic approaches to MS chiefly promote neuroprotection. A particle consisting of a watersoluble fullerene derivative functionalized with an $N$-methyl-D-aspartate receptor antagonist as an antioxidant with antiexcitotoxic potential reduced MS disease progression and myeloid cellular infiltrates in disease models [42].

Taken altogether, nanoneuromedicine is certainly composed of many divergent fields of investigation that involve the development of distinct nanoformulations targeting specific cells and sites of neural injury. Moreover, how best to traverse the $\mathrm{BBB}$ and, thus, facilitate drug payload entry to their action sites are notable. Nanotoxicology and diagnostic applications are further notable for a rapidly growing field and one that is a subject of significant interdisciplinary science.

Investigators speaking at the 2014 ASNM conference are those who study each of these parts of nanoneuroscience and are at the cutting edge of the field. They are or will soon be able to apply such technologies to facilitate improvements in disease outcomes. This is based on better ingress of contrast and therapeutic agents across the BBB, as well as in modifying drug therapeutic indices. The application of such technologies for a broad range of diseases also faces new regulatory and ethical challenges. The ASNM is humbled to bring the field to center stage and highlight the range of efforts and applications currently underway at the international stage. We are in an age of change and one where technologies will meet the needs of growing and changing populations and norms. Nanoneuromedicine will be one instrument in such a change and one poised to embrace current and future needs with real long-term solutions.

\section{Acknowledgements}

The authors would like to thank the active board members of the American Society for Nanomedicine, which includes EH Chang (acting president), R Bawa (secretary/treasurer), $M$ Foldvari (communication director), LP Balogh, $K$ Wang, M Bradbury, W Chiu, O Farokhzad and $G$ Lanza, for their continuous support, encouragement and leadership. The authors would also like to thank $R$ Taylor and L Reichardt for their support in coordinating the meeting events.

Financial \& competing interests disclosure

This work was supported by the Carol Swarts Neuroscience Research Laboratory, the Frances and Louie Blumkin Foundation, the Vice Chancellor's office of the University of Nebraska Medical Center, Seton Hall University, ViiV 
Healthcare and NIH grants 1R13NS083315-01, P01 DA028555, R01 NS36126, P01 NS31492, 2R01 NS034239, P01 MH64570 and PO1 NS43985. The authors have no other relevant affliations or financial involvement with any organization or entity with a financial interest in or financial conflict with the subject matter or materials discussed in the manuscript apart from those disclosed.

No writing assistance was utilized in the production of this manuscript.

\section{References}

1 Kanwar JR, Sun X, Punj V et al. Nanoparticles in the treatment and diagnosis of neurological disorders: untamed dragon with fire power to heal. Nanomedicine 8(4), 399-414 (2012).

2 Wong HL, Wu XY, Bendayan R. Nanotechnological advances for the delivery of CNS therapeutics. Adv. Drug Deliv. Rev. 64(7), 686-700 (2012).

3 Urakami T, Kawaguchi AT, Akai S et al. In vivo distribution of liposome-encapsulated hemoglobin determined by positron emission tomography. Artif. Organs 33(2), 164-168 (2009).

4 Marsh JN, Senpan A, Hu G et al. Fibrintargeted perfluorocarbon nanoparticles for targeted thrombolysis. Nanomedicine (Lond.) 2(4), 533-543 (2007).

5 Shapiro EM, Skrtic S, Koretsky AP. Sizing it up: cellular MRI using micron-sized iron oxide particles. Magn. Reson. Med. 53(2), 329-338 (2005).

6 Kanwar JR, Sriramoju B, Kanwar RK. Neurological disorders and therapeutics targeted to surmount the blood-brain barrier. Int. J. Nanomedicine. 7, 3259-3278 (2012).

7 Chertok B, David AE, Yang VC. Brain tumor targeting of magnetic nanoparticles for potential drug delivery: effect of administration route and magnetic field topography. J. Control Release 155(3), 393-399 (2011)

8 Yang B, Cai H, Qin W et al. Bcl-2functionalized ultrasmall superparamagnetic iron oxide nanoparticles coated with amphiphilic polymer enhance the labeling efficiency of islets for detection by magnetic resonance imaging. Int. J. Nanomedicine. 8 , 3977-3990 (2013).

9 Nel A, Swindells S, Bronich T, Gendelman HE. Nanomedicine and the fight against HIV/AIDS. Nanomedicine 9(2), 1-14 (2014).

10 Manickam DS, Brynskikh AM, Kopanic JL et al. Well-defined cross-linked antioxidant nanozymes for treatment of ischemic brain injury. J. Control Release 162(3), 636-645 (2012).

11 Batrakova EV, Gendelman HE, Kabanov AV. Cell-mediated drug delivery. Expert Opin Drug Deliv. 8(4), 415-433 (2011).
12 Mayeux R. Epidemiology of neurodegeneration. Annu. Rev. Neurosci. 26, 81-104 (2003).

13 Watson D, Castano E, Kokjohn TA et al. Physicochemical characteristics of soluble oligomeric Abeta and their pathologic role in Alzheimer's disease. Neurol. Res. 27(8), 869-881 (2005).

14 Singh S, Singh M, Gambhir IS. Nanotechnology for Alzheimer's disease detection. Dig. J. Nanomater. Bios. 3(2), 75-79 (2008)

15 Lee JH, Kang DY, Kim SU, Yea CH, Oh BK, Choi JW. Electrical detection of beta-amyloid (1-40) using scanning tunneling microscopy. Ultramicroscopy 109(8), 923-928 (2009).

16 Vestergaard M, Kerman K, Kim DK, Ha $\mathrm{MH}$, Tamiya E. Detection of Alzheimer's tau protein using localised surface plasmon resonance-based immunochip. Talanta $74(4)$, 1038-1042 (2008).

17 Yang J, Wadghiri YZ, Hoang DM et al. Detection of amyloid plaques targeted by USPIO-Abeta1-42 in Alzheimer's disease transgenic mice using magnetic resonance microimaging. Neuroimage 55(4), 1600-1609 (2011).

18 Feng L, Long HY, Liu RK et al. A quantum dot probe conjugated with abeta antibody for molecular imaging of Alzheimer's disease in a mouse model. Cell. Mol. Neurobiol. 33(6), 759-765 (2013).

19 Cabaleiro-Lago C, Quinlan-Pluck F, Lynch I et al. Inhibition of amyloid beta protein fibrillation by polymeric nanoparticles. J. Am. Chem. Soc. 130(46), 15437-15443 (2008).

20 Podolski IY, Podlubnaya ZA, Kosenko EA et al. Effects of hydrated forms of $\mathrm{C}_{60}$ fullerene on amyloid 1-peptide fibrillization in vitro and performance of the cognitive task. J. Nanosci. Nanotechnol. 7(4-5), 1479-1485 (2007).

21 Triulzi RC, Dai Q, Zou J et al. Photothermal ablation of amyloid aggregates by gold nanoparticles. Colloids Surf. B Biointerfaces 63(2), 200-208 (2008).

22 Wilson B, Samanta MK, Santhi K, Sampath Kumar KP, Ramasamy M, Suresh B. Significant delivery of tacrine into the brain using magnetic chitosan microparticles for treating Alzheimer's disease. J. Neurosci. Methods 177(2), 427-433 (2009).
23 Dauer W, Przedborski S. Parkinson's disease: mechanisms and models. Neuron 39(6), 889-909 (2003).

24 An Y, Tang L, Jiang X et al. A photoelectrochemical immunosensor based on Au-doped $\mathrm{TiO}_{2}$ nanotube arrays for the detection of alpha-synuclein. Chemistry 16(48), 14439-14446 (2010).

25 Yu J, Lyubchenko YL. Early stages for Parkinson's development: alpha-synuclein misfolding and aggregation. J. Neuroimmune Pharmacol. 4(1), 10-16 (2009).

26 Baron R, Zayats M, Willner I. Dopamine-, I-DOPA-, adrenaline-, and noradrenalineinduced growth of Au nanoparticles: assays for the detection of neurotransmitters and of tyrosinase activity. Anal Chem. 77(6), 1566-1571 (2005).

27 Santos SG, Santana JV, Maia FF Jr et al. Adsorption of ascorbic acid on the $\mathrm{C}_{60}$ fullerene. J. Phys. Chem. B 112(45), 14267-14272 (2008).

28 Klyachko NL, Haney MJ, Zhao Y et al. Macrophages offer a paradigm switch for CNS delivery of therapeutic proteins. Nanomedicine (Lond.) doi:10.2217/nnm.13.115 (2013) (Epub ahead of print).

29 Esposito E, Fantin M, Marti M et al. Solid lipid nanoparticles as delivery systems for bromocriptine. Pharm. Res. 25(7), 1521-1530 (2008).

30 Trapani A, De Giglio E, Cafagna D et al. Characterization and evaluation of chitosan nanoparticles for dopamine brain delivery. Int . J. Pharm. 419(1-2), 296-307 (2011).

31 Huang R, Ke W, Liu Y et al. Gene therapy using lactoferrin-modified nanoparticles in a rotenone-induced chronic Parkinson model. J. Neurol. Sci. 290(1-2), 123-130 (2010).

32 Hasadsri L, Kreuter J, Hattori H, Iwasaki T, George JM. Functional protein delivery into neurons using polymeric nanoparticles. J. Biol. Chem. 284(11), 6972-6981 (2009).

33 Mitchell JD, Borasio GD. Amyotrophic lateral sclerosis. Lancet369(9578), 2031-2041 (2007).

34 Hong S, Choi I, Lee S, Yang YI, Kang T, Yi J. Sensitive and colorimetric detection of the structural evolution of superoxide dismutase with gold nanoparticles. Anal Chem. 81(4), 1378-1382 (2009).

35 Reddy MK, Wu L, Kou W, Ghorpade A, Labhasetwar V. Superoxide dismutase-loaded 
PLGA nanoparticles protect cultured human neurons under oxidative stress. Appl. Biochem. Biotechnol. 151(2-3), 565-577 (2008).

36 Ali SS, Hardt JI, Dugan LL. SOD activity of carboxyfullerenes predicts their neuroprotective efficacy: a structure-activity study. Nanomedicine 4(4), 283-294 (2008).

37 Bondi ML, Craparo EF, Giammona G, Drago F. Brain-targeted solid lipid nanoparticles containing riluzole: preparation, characterization and biodistribution. Nanomedicine (Lond.) 5(1), 25-32 (2010).
38 Machtoub L, Bataveljic D, Andjus PR. Molecular imaging of brain lipid environment of lymphocytes in amyotrophic lateral sclerosis using magnetic resonance imaging and SECARS microscopy. Physiol. Res. 60(Suppl. 1), S121-S127 (2011).

39 Korn T. Pathophysiology of multiple sclerosis. J. Neurol. 255(Suppl. 6), 2-6 (2008).

40 van Kasteren SI, Campbell SJ, Serres S, Anthony DC, Sibson NR, Davis BG. Glyconanoparticles allow pre-symptomatic
In vivo imaging of brain disease. Proc. Natl Acad. Sci. USA 106(1), 18-23 (2009).

41 McAteer MA, Sibson NR, von Zur Muhlen $\mathrm{C}$ et al. In vivo magnetic resonance imaging of acute brain inflammation using microparticles of iron oxide. Nat. Med. 13(10), 1253-1258 (2007).

42 Basso AS, Frenkel D, Quintana FJ et al. Reversal of axonal loss and disability in a mouse model of progressive multiple sclerosis. J. Clin. Invest. 118(4), 1532-1543 (2008). 